\title{
Firing Patterns of Type II Spiral Ganglion Neurons In Vitro
}

\author{
Michael A. Reid, Jacqueline Flores-Otero, and Robin L. Davis \\ Department of Cell Biology and Neuroscience, Rutgers University, Nelson Laboratories, Piscataway, New Jersey 08854-8082
}

Type I and type II spiral ganglion neurons convey auditory information from the sensory receptors in the cochlea to the CNS. The numerous type I neurons have been extensively characterized, but the small population of type II neurons with their unmyelinated axons are undetectable with most recording methods. Despite the paucity of information about the type II neurons, it is clear that they must have a significant role in sound processing because they innervate the large number of outer hair cells that are critical for maintaining normal responses to stimuli. To elucidate the function of type II neurons, we have developed an approach for studying their electrophysiological features in vitro.

Type II neurons obtained from postnatal day 6-7 mice displayed distinctly different firing properties than type I neurons. They showed slower accommodation, lower action potential thresholds, and more prolonged responses to depolarizing current injection than the type I neurons. These differences were most evident in neurons from the basal, high-frequency region of the cochlea. The basal type I neurons displayed uniformly fast firing features, whereas the basal type II neurons showed particularly slow accommodation and responses to depolarization. Interestingly, neurons from the apical, low-frequency region of the cochlea showed the opposite trend. These data suggest that the type I and type II neurons have specialized electrophysiological characteristics tailored to their different roles in auditory signal processing. In particular, the type II neuron properties are consistent with cells in other sensory systems that receive convergent synaptic input for high-sensitivity stimulus detection.

Key words: cochlea; auditory; firing patterns; primary auditory nerve; accommodation; action potential; afferent; antibody; auditory; patch clamp

\section{Introduction}

Neural processing of auditory information in mammals begins with the type I and type II spiral ganglion neurons that compose the first neural elements in the auditory pathway. Although a wealth of information has been obtained about the type I neurons, the type II neurons have eluded physiological characterization because of their sparse distribution and small size. Determining the functional role of the type II spiral ganglion neurons, however, is central to our understanding of sound encoding in the auditory system. By using a combination of electrophysiology and immunochemistry, we have obtained the first recordings from this population of cells that provide insights into their function.

The primary auditory afferents display distinctive connections with hair cell receptors in the cochlea. Type I neurons compose $\sim 95 \%$ of the ganglion and innervate the inner hair cells in a one-to-one manner. Type II neurons, which compose only 5\% of the ganglion, innervate the three rows of outer hair cells (Spoendlin, 1973; Perkins and Morest, 1975; Ryugo, 1992). Intense electrophysiological analysis has led to the well accepted view that the type I neurons are responsible for the fine timing

Received Aug. 23, 2003; revised Nov. 18, 2003; accepted Nov. 19, 2003.

This work was supported by the National Institutes of Health-National Institute on Deafness and Other Communication Disorders (01856). We thank Dr. Mark R. Plummer for helpful discussions and for critically reading an earlier version of this manuscript, and Dr. Lucy Hsu for expert technical support.

Correspondence should be addressed to Dr. Robin L. Davis, Department of Cell Biology and Neuroscience, Rutgers University, Nelson Laboratories, 604 Allison Road, Piscataway, NJ 08854-8082. E-mail: rldavis@rci.rutgers.edu. D0I:10.1523/JNEUROSCI.3923-03.2004

Copyright $\odot 2004$ Society for Neuroscience $\quad$ 0270-6474/04/240733-10\$15.00/0 and frequency resolution of the auditory system. The role of the type II neurons, however, is currently unknown. Although in vivo recordings from type II neurons have been attempted, the small diameter of their unmyelinated axons makes these experiments extraordinarily difficult. Despite numerous attempts, to date only a single recording has been obtained from a positively identified type II neuron (Robertson, 1984; Brown, 1994; Robertson et al., 1999). Thus, the physiological characteristics of type II neurons require further investigation using different approaches.

The experiments presented herein focus on obtaining recordings from spiral ganglion neurons in vitro. The challenge was to identify unequivocally type II neurons without the aid of their connections to peripheral receptors and to do so for neurons that compose only $\sim 5 \%$ of the spiral ganglion. Our approach was to record from neurons that were subsequently stained with an antibody to a protein that is highly enriched in type II neurons (Hafidi, 1998; Mou et al., 1998). Thus, we have undertaken this study with the understanding that literally hundreds of recordings must be made to obtain even a limited number of type II recordings.

By taking this approach we reestablished that type I spiral ganglion neurons isolated from the apex and base of the cochlea systematically differed in their endogenous firing patterns (Adamson et al., 2002a,b). We also showed that any heterogeneity found in the population of neurons isolated from the base of the cochlea could be accounted for by the presence of the basal type II neurons. The heterogeneity that typifies neurons isolated from the apex of the cochlea, however, could not be fully accounted for by the presence of type II spiral ganglion neurons, confirming the 
relative diversity of type I apical neurons as compared with type I basal neurons. Interestingly, apical and basal type II neurons showed gradations in their electrophysiological features that were opposite to that of the type I neurons. Thus, we have begun to elucidate the electrophysiological characteristics of type II neurons and can begin to incorporate their specific firing features into our understanding of cochlear function.

Portions of these results have been published previously in abstract form (Reid and Davis, 2002, 2003).

\section{Materials and Methods}

Tissue culture. Experiments were performed on CBA/CaJ mouse spiral ganglion neurons. The spiral ganglion was removed from postnatal day (P) 6-7 animals. At this time, synaptogenesis of the spiral ganglion neurons with their peripheral targets is complete (Pujol et al., 1998), although the cartilage of the osseous spiral lamina has not yet solidified into bone. This procedure produced the best compromise between working with relatively mature cells while still retaining viability in tissue culture for detailed electrophysiological analysis. Postnatal animals were decapitated, and both inner ears were removed from the base of the cranium. Cochleas were extracted from the outer bony labyrinth. The outer ligament/stria vascularis and organ of Corti were dissected away from the central core of the cochlea that contained the spiral ganglion.

For all of the electrophysiological recordings and some of the immunocytochemistry experiments, the nerve was divided into thirds, and the base and apical sections were plated as explants in culture dishes coated with poly-L-lysine. In a limited number of experiments the entire spiral ganglion was placed in a culture dish along with markers etched into the plastic culture dish indicating the apical and basal ends of the tissue. This allowed us to assess the extreme regions of the tissue separately with staining procedures. Cells were kept at $37^{\circ} \mathrm{C}$ in a humidified incubator with $5 \% \mathrm{CO}_{2}$ and maintained in growth medium composed of DMEM supplemented with $10 \%$ fetal bovine serum, $4 \mathrm{~mm}$ L-glutamine, and $0.1 \%$ penicillin-streptomycin.

Electrophysiology. The whole-cell configuration of the patch-clamp technique was used to obtain current-clamp recordings from spiral ganglion neurons in vitro. Electrodes were pulled on a two-stage vertical puller (PP-83; Narishige, Tokyo, Japan), and the shafts were coated with Sylgard (Dow Corning) to reduce capacitance. Just before use, electrode tips were fire-polished (MF-83 microforge; Narishige); electrode resistances typically ranged from 3 to $5 \mathrm{M} \Omega$ in standard pipette and bathing solutions (see below). Pipette offset current was zeroed immediately before contacting the cell membrane. Current-clamp measurements were made with the $I_{\text {fast }}$ circuitry of the Axon Instruments (Foster City, CA) Axopatch 200A amplifier to reduce or eliminate oscillations that may occur during fast afterhyperpolarizations (Magistretti et al., 1996).

A standard set of solutions was used to approximate physiological conditions. The basic internal solution was as follows (in $\mathrm{mM}$ ): $112 \mathrm{KCl}$, $2 \mathrm{MgCl}_{2}, 0.1 \mathrm{CaCl}_{2}, 11$ EGTA, 10 HEPES-NaOH, pH 7.45. In some recordings, 5\% Lucifer yellow [dipotassium salt; Sigma L-0144 (Sigma, St. Louis, MO)] was added to the internal solution. Neurons were exposed to the following bath solution (in $\mathrm{mM}$ ): $137 \mathrm{NaCl}, 5 \mathrm{KCl}, 1.7 \mathrm{CaCl}_{2}$, $1 \mathrm{MgCl}_{2}, 17$ glucose, 50 sucrose, 10 HEPES-NaOH, pH 7.45. The osmolarity of the solution was adjusted with sucrose to $350 \mathrm{mOsm}$ to match the osmolarity of the growth medium.

Recordings were made at room temperature $\left(19-22^{\circ} \mathrm{C}\right)$ from the neuronal cell somata. Data were digitized with an Indec IDA 15125 interface in an IBM-compatible personal computer; the programs for data acquisition and analysis were written in Borland $\mathrm{C}^{++}$and Microsoft Visual Basic (generously contributed by Dr. Mark R. Plummer, Rutgers University). Each segment of data was digitized at $5 \mathrm{kHz}$ and filtered at $1 \mathrm{kHz}$. Current-clamp recordings were considered acceptable when they met the following criteria: stable membrane potentials, low noise levels, discernible membrane time constant on step current injection, and overshooting action potentials. If any of these parameters changed during an experiment, indicating compromised cell health or metabolic failure, the remaining data were not analyzed. To determine the time course of dif- ferent parameters within the voltage recordings, single exponentials were fitted using least squares.

Antibodies. Monoclonal $\beta$-tubulin (Covance, B-TUJ1, MMS-435P; 1:350 dilution) and monoclonal neurofilament-200 (Sigma, N-0142; 1:100 dilution) antisera were used to distinguish neurons and processes from background satellite cells. Peripherin antibody was used to distinguish type II neurons from the total population of spiral ganglion neurons. On the basis of results reported in vivo (Hafidi, 1998) and confirmed in vitro (Mou et al., 1998), this intermediate filament has been found to be enriched in type II spiral ganglion neurons. Monoclonal (MAB 1527; Chemicon, Temecula, CA) and polyclonal (AB-1530; Chemicon) peripherin antisera were used in the following dilutions: 1:75 and 1:4000, respectively.

The Kv1.1 subunit of the Shaker family was also evaluated in this study. The Kv1.1 polyclonal antibody (Alomone Labs, APC-009; 1:25 dilution) was made against amino acid residues 416-495 of the C terminus of mouse full-length Kv1.1 protein. Western blotting and immunoprecipitation assays showed that reaction products were specific for the Kv1.1 subunit-purified antibodies, and no cross-reactivity occurred between mKv1.2 or mKv1.3, the two most closely related voltage-gated $\mathrm{K}^{+}$ channel proteins of the Shaker-like subfamily (Koch et al., 1997).

Immunofluorescence. Indirect immunofluorescence was used on cultures fixed in $100 \%$ methanol at $-20^{\circ} \mathrm{C}$ for $6 \mathrm{~min}$. Cultures were then rinsed thoroughly and incubated at room temperature for 1 additional hour in $5 \%$ normal goat serum. The primary antibody was applied and left for $1 \mathrm{hr}$ at room temperature or for $24-48 \mathrm{hr}$ at $4^{\circ} \mathrm{C}$. A fluorescentconjugated secondary antiserum was subsequently applied for $1 \mathrm{hr}$ at room temperature. Between each step, except after the application of the blocking solution, the tissue was rinsed three times for 5 min with $0.01 \mathrm{M}$ PBS, pH 7.4.

At the conclusion of the electrophysiological experiments, neurons were typically stained with polyclonal anti-peripherin followed by a tetramethylrhodamine isothiocyanate (TRITC)-conjugated anti-rabbit secondary antibody (Sigma, T-6778; 1:100 dilution) to specifically label type II spiral ganglion neurons. In some cases the full population of neurons was identified by applying monoclonal neurofilament-200 antibody followed by FITC-conjugated anti-mouse secondary antibody (Sigma, F-4018; 1:100 dilution).

To compare Kv1.1 antibody staining patterns in identified type I and II neurons, we devised a triple-staining protocol that used two monoclonal antibodies (peripherin and $\beta$-tubulin, both from mice) and one polyclonal antibody (Kv1.1, from rabbit). The order of monoclonal antibody application was critical to retain the specific staining of the peripherin antibody. For this reason, the peripherin antibody was always applied first and followed by a TRITC-conjugated anti-mouse secondary antibody (Sigma, T-7782; 1:100 dilution). The anti- $\beta$-tubulin primary antibody was applied next followed by its Alexa-Fluor-conjugated anti-mouse secondary antibody (Molecular Probes, A-11045; 1:100 dilution). In this case the Alexa-Fluor secondary antibody would be expected to bind not only to the $\beta$-tubulin antibody, but also to any unbound peripherin antibody. Because we were only using $\beta$-tubulin antibody as a marker for all neurons, this possible double labeling of the type II neurons was not detrimental to interpretation of the staining patterns. Last, Kv1.1 polyclonal antibody application was followed by FITC-conjugated anti-rabbit secondary antibody (Sigma, F-0511; 1:100 dilution). The secondary antibody used to demarcate the ion channel subunit did not interact with the monoclonal antibodies that were used previously. Therefore, for our immunocytochemical analysis of Kv1.1 staining patterns, we were able to observe the Kv1.1 specific staining (green fluorescence) in the full complement of neurons (blue fluorescence) as well as within the specifically labeled type II neurons (red fluorescence).

Preadsorption experiments performed for Kv1.1 antibody, reported previously for the antibodies used in this study (Adamson et al., 2002b), showed that staining was abolished by preincubation of the antisera with the appropriate synthetic peptide. Additionally, negative controls, where the primary antibody was omitted, were routinely evaluated to assay the specificity of all of the secondary antibodies. No specific labeling was detected in any of these control experiments. Another test of antibody specificity was to use more than one antibody for the same ion channel subunit, because there would be little likelihood that nonspecific staining 


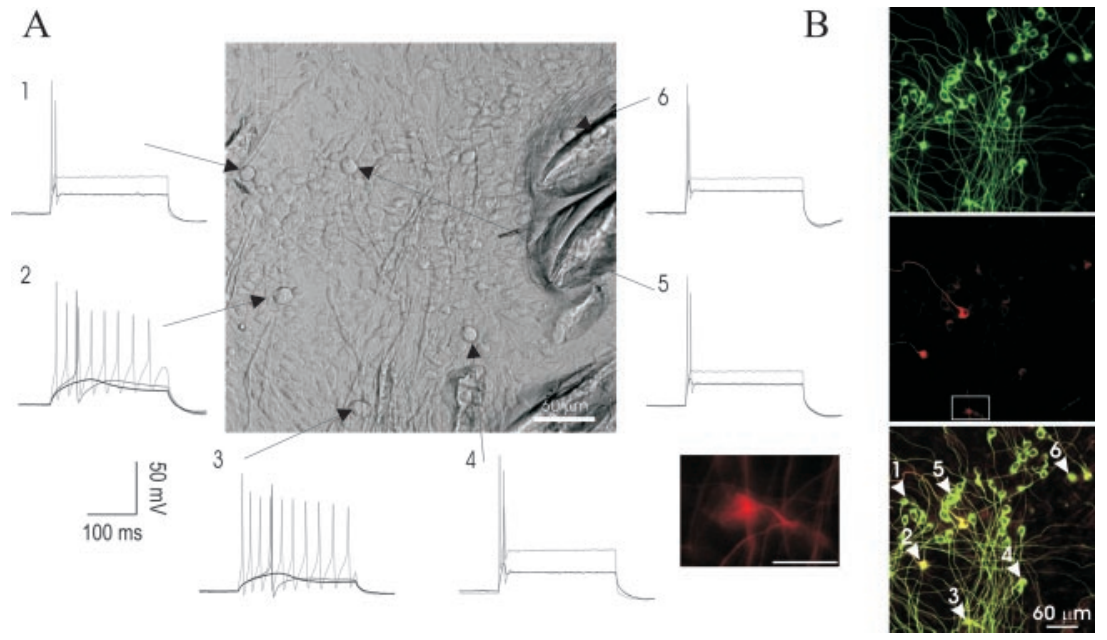

Figure 1. Putative basal type II neurons show electrophysiological response properties that are distinctly different from basal type I neurons. $A$, Current-clamp traces from the neurons indicated (arrows) in the Hoffman image at the center of the panel. For this and subsequent figures, three sample responses to a 240 msec step depolarization are shown for each neuron: the bottom trace was subthreshold, the middle trace was taken at or close to electrophysiological threshold, and the top trace was at a suprathreshold level to show the maximum number of action potentials fired by a particular neuron. Neurons were isolated from the basal cells of P7 CBA/CaJ mice and maintained in standard culture conditions for $6 \mathrm{~d}$. $B$, Anti-peripherin labeling reveals presumptive type II neurons. The same field shown in $A$ stained with different antibodies. Top panel, Monoclonal neurofilament 200; middle panel, polyclonal peripherin; bottom panel, superimposed images of neurofilament 200 and peripherin. Inset, High magnification of neuron 3 stained with peripherin.

would be identical in differently prepared antibodies. The staining patterns that we observed in spiral ganglion neurons did not differ between the different antisera made to the Kv1.1 channel subunit, thus giving us confidence that the antibodies selectively labeled the ion channel subunits that they were made against (Adamson et al., 2002b). The optimal concentration of the Kv1.1 ion channel antibody used was determined with serial dilutions and by choosing a range of concentrations that were high enough to detect the neuronal staining and low enough to keep the background staining levels to a minimum.

Image acquisition and quantification of immunofluorescent data. Images were acquired with a Hamamatsu C4742-95 charge-coupled digital camera using Zeiss Axiovision software and further processed in Adobe Photoshop. Brightness, contrast, and sharpening tools in Adobe Photoshop were used to enhance the contrast between neuronal and background signals. Comparisons of staining were made only between images acquired with identical exposure times that received the same digital enhancements.

Analysis of antibody staining in neurons isolated from the apical and basal cochlea was accomplished by photographing regions that contained both type I and type II spiral ganglion neurons. Each neuron in the photograph was evaluated according to staining intensity by quantifying average pixel intensity (Adobe Photoshop) from three separate measurements in the most intensely stained region of the neuron. Four background measurements surrounding each cell were also averaged and then subtracted from the somata luminance measurement to adjust for the surrounding nonspecific staining. We obtained similar results from two different Kv1.1 antibody concentrations, 1:50 and 1:25; however, because the higher concentration was selected for most of the experiments, we limited our analysis to 1:25. Statistical comparisons were made using Student's two-tailed $t$ test.

\section{Results}

Type II neurons have small to average-size somata that, in mouse, are either bipolar or pseudomonopolar in shape (Perkins and Morest, 1975; Berglund and Ryugo, 1987; Romand and Romand, 1987), but on the basis of their size and shape alone, they cannot be identified unequivocally when separated from the sensory receptors. Type II neurons, however, possess molecular markers that can be used to distinguish them from type I neurons. We have shown previously that antibodies to the neurofilament protein peripherin label type II neurons preferentially in vitro (Mou et al., 1998). We also observed recently that two of the four family members of the membrane-associated guanylate kinases (MAGUKs), synapse-associated protein (SAP) and SAP102, are enriched in the type II spiral ganglion neurons (Hsu et al., 2002). MAGUKs not only participate in targeting of synaptic proteins, but they also associate with specific groups of ion channels that bind to their PSD-95/Dlg/ ZO-1 (PDZ) domain, such as Shaker and inward rectifier $\mathrm{K}^{+}$channels (Fanning and Anderson, 1999). This result is consistent with the idea that type II neurons may possess endogenous electrical features that differ from the type I spiral ganglion neurons. With these markers in hand, we made whole-cell current-clamp recordings from spiral ganglion neurons of known location in the culture dish so that they could be subsequently stained with peripherin antibody for classification.

A total of 228 recordings were obtained using this method, from which 9 type II neurons were unequivocally identified. Results from recordings of basal type II neurons will be presented first, along with a comparison of basal type II and type I neurons. This will be followed by a description of apical type I neurons and an analysis of the staining patterns of the Kv1.1 $\alpha$-subunit in basal and apical type II neurons.

\section{Basal type II spiral ganglion neurons}

With few exceptions, spiral ganglion neurons isolated from the base of the cochlea possess uniform firing features (Adamson et al., 2002a,b). In response to suprathreshold depolarization, these neurons tend to fire almost instantaneously with brief duration action potentials that accommodate rapidly. On rare occasions, however, we encountered neurons that were substantially different from the "fast" firing features that typified the basal type I neurons. We therefore set out to test the hypothesis that this firing pattern could be attributed to the type II spiral ganglion neurons.

Our initial strategy was simply to record from as many identified neurons as possible in each culture dish. Before fixation and immunocytochemical processing, a map was drawn from the image at $40 \times$ (Hoffman optics) to distinguish each recorded cell for subsequent evaluation. This approach had the additional advantage of allowing direct comparisons to be made between type I and II neurons that were isolated from a restricted region of the cochlea (Fig. 1). In the example shown, two of the six basal spiral ganglion neurons from which recordings were made stained positively for peripherin (Fig. $1 B$ ). The firing patterns obtained from these putative type II spiral ganglion neurons (Fig. $1 A$, cells 2 and 3 ) were strikingly different from that observed from their type I counterparts (Fig. 1A, cells 1, 4-6). Basal type II neurons exhibited significantly slower subthreshold onset kinetics $(\tau=26.2 \pm$ 1.7 vs $6.4 \pm 1.6 \mathrm{msec} ; p<0.01$ ) that were measured from single exponentials fitted to subthreshold onset response (Fig. $1 \mathrm{~A}$, bottom traces). They showed longer action potential latencies $(84.5 \pm 2.5$ vs $10.0 \pm 0.73 \mathrm{msec} ; p<0.01)$ and durations $(2.1 \pm$ 

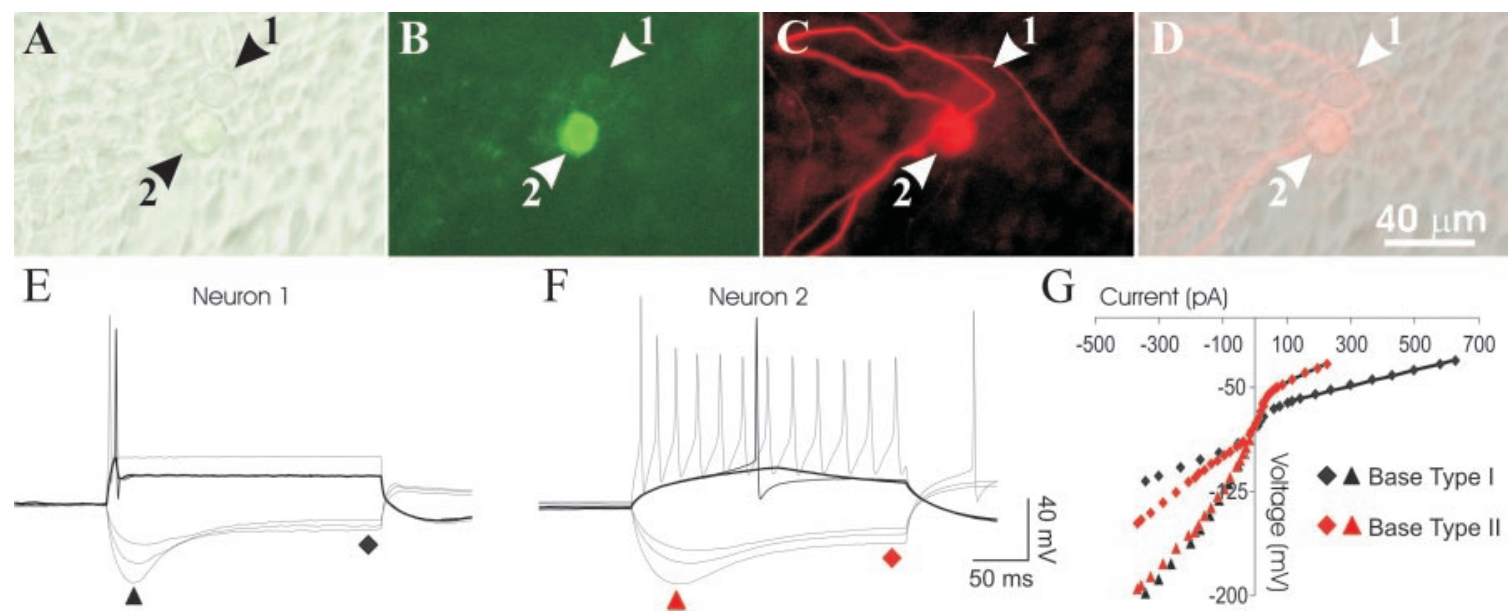

G

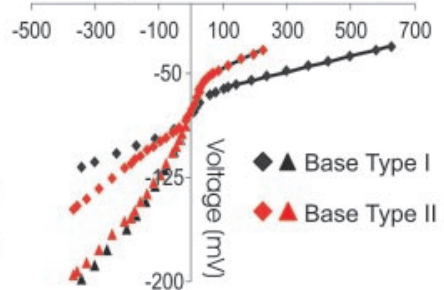

Figure 2. Basal type I and type II neurons located side by side in vitro differed in their endogenous firing features. Lucifer yellow was included in the internal solution for one of the recordings to identify unequivocally the neuron from which the recording was made. $A-D$, Images of the same field in vitro are shown for different combinations of illumination and filter settings. $A$, Incandescent illumination with Hoffman optics shows the general shape of the neurons on a background of satellite cells. The two neurons from which recordings were made are labeled 1 and 2. B, Fluorescent image of the Lucifer yellow-filled neuron (green), labeled 2. C, Fluorescent image showing that polyclonal peripherin antibody (red) is enriched in the neuron labeled 2 and is essentially absent from the neuron labeled 1.D, Combination of images shown in $A$ and $C$ to show the location of the peripherin-positive neuron. E, Current-clamp recording from neuron 1 shows firing features typical of a type I basal spiral ganglion neuron. F, Current-clamp recordings from neuron 2. Calibration bar applies to $E$ and F. G, Current-voltage relationships are shown for the type I (black) and type II (red) neurons. Measurements were made at the end of the $240 \mathrm{msec}$ step depolarization (diamonds) for both depolarizing and hyperpolarizing constant-current injections. The peak voltages achieved in response to hyperpolarizing constant-current injections were denoted with triangles.

0.3 vs $1.12 \pm 0.05 \mathrm{msec} ; p<0.05$ ) at threshold (Fig. $1 A$, middle traces) and slower accommodation [measured as the maximum number of action potentials fired to a $240 \mathrm{msec}$ step depolarization $\left(A P_{\max } ; 9 \pm 1\right.$ vs $1.2 \pm 0.2$ spikes; $p<0.01$ ) (Fig. $1 A$, top traces). Furthermore, the type II neuron threshold voltages were significantly more negative than the neighboring type I voltages $(-65.3 \pm 1.65$ vs $-60.5 \pm 0.9 \mathrm{mV} ; p<0.05)$. These were all striking differences between the two categories of cells.

Experiments of the kind described above yielded a total of three recordings from putative basal type II neurons. To confirm these observations, we used an alternate strategy in which Lucifer yellow was included in the recording pipette solution, and wholecell current-clamp recordings were made from a single neuron or pairs of neurons in each culture dish. The purpose of these experiments was to guarantee that a neuronal recording could be associated unequivocally with an immunocytochemical profile. With this method we were able to find and characterize two additional peripherin-positive basal neurons. In the example shown (Fig. 2), even neurons located side by side showed profoundly different firing features. The neuron labeled 1 (Fig. 2A-D) showed rapid accommodation $\left(A P_{\max }=1\right)$, fast subthreshold onset kinetics $(\tau=5.1 \mathrm{msec})$, and abbreviated action potential latency (13.1 $\mathrm{msec})$ at its electrophysiological threshold $(-50.4 \mathrm{mV})$ (Fig. 2 E). These fast firing features, typical of a basal type I spiral ganglion neuron, differed substantially from the neighboring Lucifer yellow-filled neuron (Fig. $2 A, B$ ) that was subsequently found to be peripherin positive (Fig. $2 C, D$ ). In addition to slow accommodation $\left(A P_{\max }=12\right)$, the subthreshold onset kinetics $(\tau=38.4$ $\mathrm{msec})$ and action potential latency $(127 \mathrm{msec})$ were prolonged, and the electrophysiological threshold was more hyperpolarized $(-56.3 \mathrm{mV})$ (Fig. $2 \mathrm{~F}$ ). The action potential duration in these two examples did not differ; the time elapsed at the midpoint between the peak and nadir for each measurement was $1.5 \mathrm{msec}$. Input resistances calculated from the lines fitted to the linear portion of the voltage responses to depolarizing constant current injections (Fig. $2 G$ ) were 57.3 and $108.8 \mathrm{M} \Omega$ for the type I and II neurons, respectively.
Differences were also observed between the type I and II basal neurons in their voltage responses to hyperpolarizing constant current injections. The characteristic "sag" in the voltage observed for spiral ganglion neurons (Mo and Davis, 1997b) was slowed in the type II neuron (Fig. $2 F$ ) compared with the neighboring type I (Fig. 2E). Furthermore, the magnitude of this inward rectification was smaller for the basal type II neuron than the type I (Fig. $2 G$ ), a result that may be consistent with the more negative thresholds of type II neurons.

Not all of the putative type II basal spiral ganglion neurons showed slow accommodation. Two of the five peripherinpositive basal cells had an $A P_{\max }$ of 2 . Recordings from both of these type II cells showed latency, subthreshold kinetics, and inward rectification time courses that were substantially slower than the basal type I neurons yet relatively faster than the more slowly adapting type II basal neurons. We found a linear relationship between latency and accommodation for the type II spiral ganglion neurons; longer latencies were predictive of slower accommodation, and not surprisingly, this same relationship was also observed for onset kinetics and the time course of inward rectification when compared with $A P_{\text {max }}$ (data not shown).

\section{Quantitative comparison of base type I and type II neurons}

When all of the experiments from basal neurons were taken together we found that a relatively small proportion of neurons isolated from the basal cochlea ( 5 of a total of $83 ; \sim 6 \%$ ) were unequivocally peripherin positive. Nevertheless, they showed significant differences $(p<0.01)$ from the type I electrophysiological firing pattern in all of the parameters that we examined (Fig. 3).

Consistent with previous reports from our laboratory (Adamson et al., 2002a,b) the basal type I, peripherin-negative neurons $(n=78)$, which are responsible for coding sounds in the highfrequency range, could be consistently classified as rapidly accommodating at suprathreshold levels, firing on average a maximum of only one action potential $(1.1 \pm 0.04)$ in response to a $240 \mathrm{msec}$ step depolarization. Action potential latencies (10.8 \pm $0.44 \mathrm{msec})$ and durations $(1.25 \pm 0.03 \mathrm{msec})$ were typically ab- 

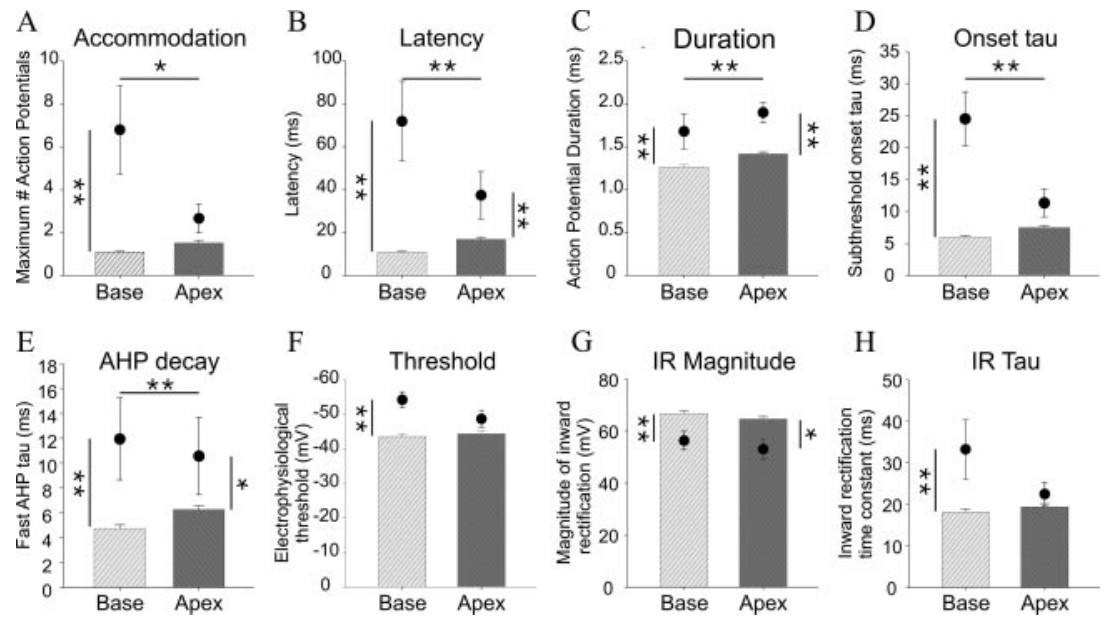

Figure 3. Electrophysiological responses of type II spiral ganglion neurons show significant differences from their type I counterparts. A, Accommodation is represented as the maximum number of action potentials that a neuron is capable of firing during suprathreshold step depolarizations 240 msec in duration. For all panels, the light gray hatched bars labeled "Base" and dark gray hatched bars labeled "Apex" represent the type I neurons, whereas the circles above each bar represent the type II neurons from the base and apex. The horizontal lines above the two bars indicate statistically significant differences between the basal and apical type I neurons, vertical lines on the left represent statistically significant differences between the base type I and type II neurons, and vertical lines on the right represent statistically significant differences between the apical type I and type II neurons. No significant differences between apical and basal type II neurons were found for any of the electrophysiological parameters measured. ${ }^{*} p<0.05 ;{ }^{* *} p<0.01$. $B$, The action potential latency, at electrophysiological threshold (the lowest voltage level at which an action potential was observed), was measured as the time from the stimulus onset to the peak of the action potential. C, Action potential duration, at electrophysiological threshold, was measured at the point halfway between the peak and nadir of the waveform. $D$, The slow component of the onset tau was measured with a single exponential fitted to the voltage change from onset of the stimulus, excluding any initial fast component, to the peak voltage. Measurements were taken from subthreshold recordings made just below electrophysiological threshold. $E$, The time course of the fast AHP decay was measured by fitting a single exponential starting just after the nadir of the action potential. $F$, The voltage level representing electrophysiological threshold was measured from the peak voltage achieved in slightly subthreshold responses to depolarizing current injection. $G$, The magnitude of inward rectification was determined by measuring the difference between the peak and the plateau voltages from hyperpolarizing responses that peaked at $-185 \pm 3 \mathrm{mV}$. $H$, The time course of the inward rectification was measured with single exponential fits to the traces that peaked at $-185 \pm 3 \mathrm{mV}$.

breviated even when measured at electrophysiological threshold (Fig. 3A-C). In contrast to this fast electrophysiological profile, the basal type II neurons fired significantly more action potentials in response to the same $240 \mathrm{msec}$ suprathreshold stimuli (6.8 \pm $2.1 ; p<0.01)$. Additionally, prolonged latencies $(71.8 \pm 18.5$ msec; $p<0.01)$ and action potential durations ( $1.68 \pm 0.2 \mathrm{msec}$; $p<0.01)$ were consistently observed at electrophysiological threshold for this cell type (Fig. $3 A-C$ ).

The distinctions between basal type I and type II spiral ganglion neurons is further exemplified by differences in the time course of onset (Fig. 3D) to depolarizing step current injections and the decay of the fast afterhyperpolarization (AHP) (Fig. 3E), measured at electrophysiological threshold with single exponential fits. In each case, the responses of basal type II neurons were slow when compared with the type I neurons. The onset tau $(24.5 \pm 4.2 \mathrm{msec})$ and fast AHP decay $(11.9 \pm 3.3 \mathrm{msec})$ for the type II subcategory were both significantly longer than the same parameters measured for the type I spiral ganglion neurons (5.6 \pm 0.25 and $4.7 \pm 0.3 \mathrm{msec}$, respectively) (Fig. $3 D, E$ ).

The basal type II neuronal thresholds were significantly more hyperpolarized $(-54.2 \pm 2.3 \mathrm{mV})$ than their type I counterparts $(-43.6 \pm 0.8 \mathrm{mV} ; p<0.01)$ (Fig. $3 F)$. Furthermore, there were significant differences in the magnitude and time course of the voltage changes produced by hyperpolarizing current injection. This was quantified by analyzing voltage responses that peaked at $-185 \pm 3 \mathrm{mV}$. This value was chosen because it was well within the linear portion of the current-voltage relationship (Fig. 2G) and because all cells studied achieved this response with our current injection protocol. The magnitude of the difference between the peak hyperpolarizing response and the plateau level (at the end of the 240 msec stimulus) was smaller for the type II $(56.4 \pm 3.5 \mathrm{mV})$ than the type I $(66.8 \pm 0.9$ $\mathrm{mV}$ ) neurons (Fig. $3 G$ ). Additionally, the time course of decay at the same voltage level was slower for the type II neurons $(\tau=33.3 \pm 7.1 \mathrm{msec})$ than the type I neurons $(\tau=18.1 \pm 0.8 \mathrm{msec})($ Fig. $3 H)$.

\section{Apical type II spiral ganglion neurons}

A total of four recordings were obtained from unequivocally identified type II apical neurons. Three of these were from P6-7 animals; the other was obtained from a P4 animal, and data from this cell are not formally included in this analysis. Nonetheless, the properties of this cell were similar to those obtained from P6-7 animals.

In two of the recordings from type II apical neurons, additional recordings were obtained from type I apical neurons in the same culture dish. Interestingly, we found that type II neurons displayed firing features that were not as slowly accommodating as neighboring apical type I neurons. This observation is shown for a set of fortuitous recordings from a pair of apical neurons, one of which was peripherin positive whereas the other was peripherin negative (Fig. 4). This enabled a direct comparison to be made between type I and II neurons isolated from the same cochlear region. The neuron filled with Lucifer yellow (Fig. 4A-D, 1) showed slow accommodation $\left(A P_{\max }=14\right)$, prolonged latency $(144.6 \mathrm{msec})$, and slow onset kinetics $(\tau=28.6 \mathrm{msec})$, similar to that observed in three of the five basal type II neurons (Fig. $4 E$ ), but because it lacked peripherin antibody labeling it was classified as an apical type I neuron. The other neuron of the pair (Fig. $4 A-D, 2)$, which was relatively more rapidly accommodating $\left(A P_{\max }=4\right)($ Fig. $4 F)$, was densely labeled with anti-peripherin, indicating that it was a type II spiral ganglion neuron. Another complete recording from an identified type I apical neuron, located outside the field of view shown in Figure 4, was rapidly accommodating, having an $A P_{\max }$ of 2. This neuron showed relatively rapid latency $(17.8 \mathrm{msec})$ and onset kinetics $(\tau=6.2 \mathrm{msec})$ as shown in Figure $4 G$. Thus, the type II apical spiral ganglion neuron showed an intermediate firing phenotype between the rapidly adapting and slowly adapting apical type I neurons. This same conclusion could also be drawn from the second experiment in which one recording of five in a single culture dish showed that the type II firing pattern was intermediate in its properties between one slowly adapting neuron and three rapidly adapting ones.

\section{Quantitative comparison of apex type I and type II neurons}

In a previous study (Adamson et al., 2002b) we described heterogeneous firing patterns in the population of spiral ganglion neurons isolated from the apical cochlea. Accommodation, for example, ranged from rapid to slow, although we found that the 


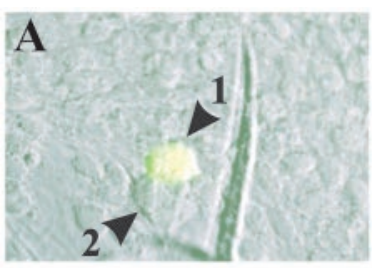

$\mathrm{E}$

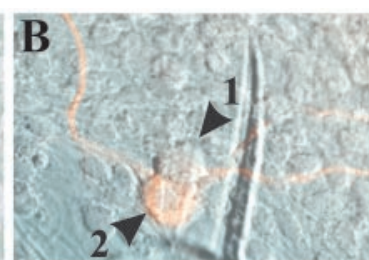

$\mathrm{F}$
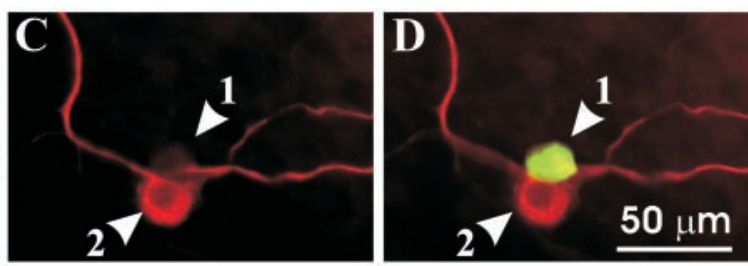

G

Apex type I (RA)

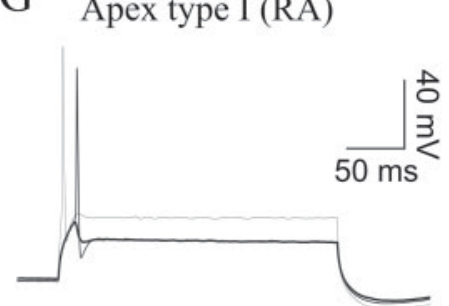

Figure 4. Apical type II neurons show features that are intermediate between the rapidly accommodating and slowly accommodating apical type I neurons. $A-D$ show combinations of fluorescent and bright-field images of two neurons from which recordings were made. A, Hoffman optics in combination with the fluorescent image of the Lucifer yellow-filled neuron show the general shape of the neurons on a background of satellite cells. The two neurons from which recordings were made are labeled 1 and 2. B, Hoffman optics in combination with peripherin antibody labeling show that the filled cell is not stained with the type Il-specific antibody. C, Fluorescent image showing that peripherin antibody (red) is enriched in the neuron labeled 2 and is essentially absent from the neuron labeled 1.D, Combination of Lucifer yellow and $\alpha$-peripherin fluorescent images to confirm that the Lucifer yellow dye is located within the peripherin-negative neuron. $E$, Current-clamp recording from neuron 1.F, Current-clamp recordings from the peripherin-positive neuron labeled 2. G, Current-clamp traces from a neuron outside of the field of view that did not stain with peripherin. The calibration bar, bottom right, applies to $E-G$.

apical spiral ganglion neurons could be placed into two subcategories on the basis of the number of action potentials that they fired in response to a $240 \mathrm{msec}$ duration step depolarization. Most of the neurons were rapidly accommodating (firing six or fewer action potentials per $240 \mathrm{msec}$ step depolarization), whereas the remaining neurons were slowly accommodating (more than six action potentials per $240 \mathrm{msec}$ step depolarization). One possible explanation was that the apical type II neurons make up this relatively small population of slowly adapting neurons. The results described above argue against this idea because all of the apical peripherin-positive neurons that we encountered fired fewer than six action potentials in response to prolonged step depolarizations.

Not unexpectedly, therefore, even with the type II neurons eliminated from the data set, we still found that $A P_{\max }(1.5 \pm 0.1$ spikes), action potential latencies (16.8 $\pm 1.1 \mathrm{msec})$, and durations ( $1.4 \pm 0.02 \mathrm{msec}$ ) for the type I apical neurons were significantly longer than basal type I measurements $(p<0.05, p<$ 0.01 , and $p<0.01$, respectively) (Fig. $3 A-C$ ). When comparing the 3 type II apical neurons with the 142 apical type I neurons, we found that some, but not all, of the firing parameters were significantly different. The $A P_{\max }$ was one feature that did not differ between the two classes of neurons in the apex. The $A P_{\max }$ for the type II neurons was higher than that found for the type I neurons, $2.7 \pm 0.7$, but it did not achieve significance $(p=0.18)$. In contrast to $A P_{\max }$, comparisons made between apical type I and II neurons for action potential latency and duration yielded significant differences for both $(p<0.01)$. Much like what had been found for the basal type II neurons, apical type II neurons showed longer latencies and durations $(37.4 \pm 11$ and $1.9 \pm 0.1 \mathrm{msec}$, respectively) than their type I counterparts.

The differences between apical type I and type II spiral ganglion neurons was not as pronounced, however, as the differences observed between basal type I and II neurons. Nevertheless, similar trends were clearly observed. For example, the mean values of the onset time constant $(11.3 \pm 2.2 \mathrm{msec})$ and AHP decay $(10.6 \pm$ $3.1 \mathrm{msec}$ ) measurements were both greater for the type II neurons compared with the type I neurons $(7.4 \pm 0.3$ and $6.3 \pm 0.3 \mathrm{msec}$, respectively) (Fig. $3 D, E$ ). The AHP decay time constant was significantly different $(p<0.05)$, and the comparison for onset time constant was close to significance $(p>0.06)$. Furthermore, the threshold level of apical type II neurons $(-48.7 \pm 2.5 \mathrm{mV})$, although more hyperpolarized than the type I neurons $(-44.4 \pm$ $0.8 \mathrm{mV}$ ), was not significantly different (Fig. $3 F$ ), nor was the time course of the inward rectification $(\tau=22.5 \pm 2.8$ and $19.4 \pm$ $0.8 \mathrm{msec}$ for the type II and type I neurons, respectively) (Fig. $3 \mathrm{H})$. Nevertheless, the magnitude of the inward rectification was significantly different at the 0.01 level $(53.1 \pm 3.8$ and $64.7 \pm 1.0$ $\mathrm{mV}$ for the type II and type I neurons, respectively) (Fig. $3 G$ ).

These results confirm the idea that the apical type II neurons do not account for the heterogeneity that one observes in the apical firing properties at the ages and times in culture that we investigated. This prompted us to evaluate electrophysiological parameters separately for the rapidly and slowly accommodating apical neurons and compare them with the apical type II neurons (Fig. 5). From this analysis we found that in addition to the latency, duration, AHP decay, and magnitude of the inward rectification, the onset time constant was also significantly different between the apical type II neurons and the rapidly adapting type I neurons (Fig. 5D). Furthermore, we found that some of the parameters that differed significantly when considering the total population of apical neurons could be attributed in large measure to the differences between the rapidly adapting type I neurons and the type II neurons (Fig. $5 C, E, F$ ). It should also be noted that even with the slowly adapting type I neurons removed from the population of apical type I neurons, significant differences were still found between these and the basal type I neurons; in some cases the level of significance increased.

Regardless of whether we consider only the rapidly accommodating apical type I neurons or the total apical population, we found that the apical type II neurons displayed relatively slow accommodation and kinetics and lower thresholds for action potential initiation. When the apical type II neurons were specifically compared with the basal type II neurons, we found a ten- 

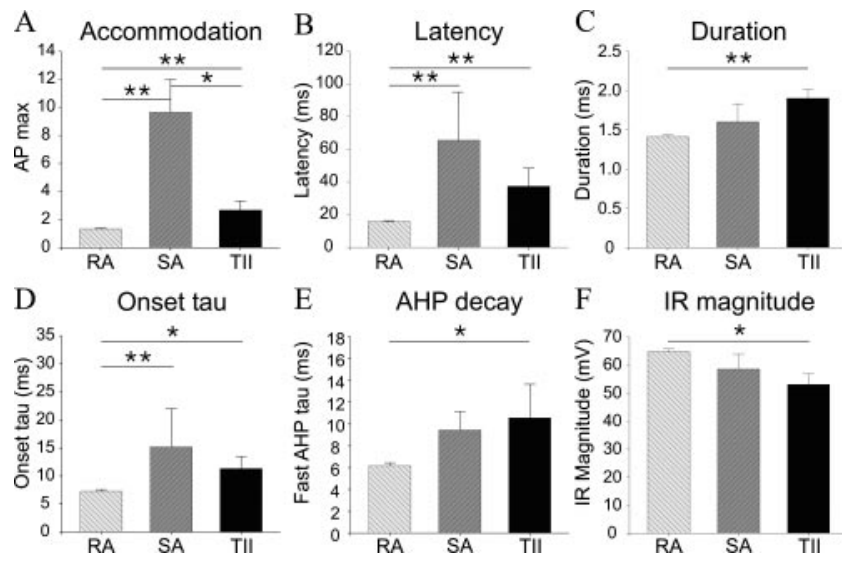

Figure 5. Comparison of the electrophysiological parameters of apical type II neurons with the rapidly and slowly accommodating categories of apical type I neurons for accommodation $(A)$, latency $(B)$, action potential duration $(C)$, subthreshold onset kinetics $(D)$, decay of the fast afterhyperpolarization $(E)$, and magnitude of inward rectification $(F)$. Measurements were identical to those described in Figure 3. RA, Rapidly accommodating; SA, slowly accommodating; TII, type II.

dency for the basal type II neurons to possess slower features. This was true for $A P_{\max }$ (Fig. $3 A$ ), latency (Fig. $3 B$ ), onset time constant (Fig. 3D), threshold (Fig. $3 F$ ), and the time course of the inward rectification (Fig. $3 H$ ). Interestingly, this is opposite to the relationship between the apical and basal type I neurons, which show slower electrophysiological parameters for the apical type I neurons. If these observed trends in the apical and basal type II electrophysiological phenotypes are borne out by additional data, it would have profound implications for how the electrophysiological phenotype is established and maintained compared with the type I neurons (Adamson et al., 2002a), as well as for its functional significance.

\section{Immunocytochemical analysis of Kv1.1 distribution in type II spiral ganglion neurons}

The unexpected observation that accommodation was apparently oppositely distributed in type I and II neurons was further studied using immunocytochemistry to determine the pattern of a well defined voltage-gated ion channel subunit in spiral ganglion neurons. This technique allows a larger population of type II spiral ganglion neurons to be examined and compared with type I neurons isolated from the same cochlear region. For these experiments spiral ganglion neurons were colabeled with peripherin- $\beta$-tubulin and Kv1.1 antibodies (see Materials and Methods). We chose to examine the Kv1.1 ion channel subunit because previous experiments from our laboratory had shown that rapidly accommodating neurons exposed to specific Kv1.1/ Kv1.2/Kv1.6 blockers [ $\alpha$-dendrotoxin (DTX) and DTX-K] became slowly accommodating (Mo et al., 2002). The Kv1.1 subunit, therefore, contributes to limiting the number of action potentials that spiral ganglion neurons can fire in response to prolonged stimuli, and their relative density should relate to the electrophysiological measurements of $A P_{\max }$.

From the electrophysiological profiles of the type II neurons, we hypothesized that the slowly accommodating type II basal neurons would possess greatly reduced $\alpha$-Kv1.1 staining intensity when compared with the surrounding basal type I neurons. This expectation was borne out by the $\alpha$-Kv1.1 staining patterns that we observed from neurons innervating the basal cochlear region. In the example shown in Figure $6 A-C$, neurons identified as type II in the basal region showed very little Kv1.1 antibody staining compared with neighboring type I neurons. Consistent with this idea, we also found that $\alpha$-Kv1.1 was highly enriched in the basal type I neurons compared with the apical type I and II neurons in sister cultures using identical immunocytochemical protocols, photographic exposure times, and contrast enhancement procedures.

For the apical neuronal cultures, we did not necessarily expect $\alpha$-Kv1.1 labeling to be uniform because of the heterogeneous accommodation found in this region. We have observed apical neurons with $A P_{\max }$ levels ranging from 1 to 26 (Mo and Davis, 1997a; Adamson et al., 2002b), whereas the $A P_{\max }$ of apical type II neurons that we observed to date ranged only from 2 to $4(n=4)$. Therefore, compared with basal type I neurons, we expected to observe diverse levels of $\alpha$-Kv1.1 in apical type I neurons with lower overall staining intensity, reflecting the heterogeneous $A P_{\text {max }}$ levels that were recorded electrophysiologically. Because of the intermediate $A P_{\max }$ levels of the apical type II neurons compared with the apical type I neurons, we expected to observe both darker and lighter staining apical type I neurons compared with the neighboring type II neurons, reflecting rapidly and slowly accommodating apical type I neurons, respectively. Moreover, direct comparisons between the apical and basal type II neurons would be expected to yield less $\alpha$-Kv1.1 staining density in the basal type II neurons than in the apical type II neurons. This predicted distribution would run counter to our observations for the population of type I neurons, having type II neurons with slower kinetics and accommodation in the high-frequency area and relatively faster electrophysiological parameters in the lowfrequency region.

Our results from the immunocytochemical analysis of the apical cultures revealed staining patterns consistent with our electrophysiological analysis of neuronal accommodation. Individual apical type I neurons displayed both higher and lower $\alpha$-Kv1.1 staining intensities than the apical type II neurons. Two examples of $\alpha$-Kv1.1 staining are shown to illustrate this result. The example shown in Figure $6 D-F$ revealed an $\alpha$-Kv1.1 staining pattern suggestive of the electrophysiological profile demonstrated from the pair of apical neurons shown in Figure $4, E$ and $F$. The slowly accommodating apical type I neuron would be expected to possess very low $\alpha-\mathrm{Kv} 1.1$ staining density compared with a neighboring apical type II neuron. On the other hand, similar to what we have observed from rapidly adapting type I apical neurons, exemplified by the traces in Figure $4 G$, type I apical neurons could also be more enriched in Kv1.1 antibody staining than nearby type II neurons (Fig. 6G-I).

To establish the significance of the staining patterns described above, quantitative measurements were obtained from a total of eight separate platings in which 37 basal type I neurons, 9 basal type II neurons, 26 apical type I neurons, and 18 apical type II neurons were analyzed (Fig. 7A). Kv1.1 antibody luminance in basal type I neurons was significantly greater than the apical type I neurons $(p<0.01)$. We also found that the basal type II neurons were significantly different from the basal type I neurons $(p<0.01)$, having less dense Kv1.1 antibody staining, thus reflecting the slower accommodation of these cells. On the other hand, when all of the apical measurements were taken together, we did not observe significant differences between the apical type I and type II neurons, most likely reflecting their intermediate phenotype between rapidly accommodating and slowly accommodating apical type I neurons $(p>0.4)$. Most importantly, however, this analysis allows us to make comparisons between greater numbers of apical and basal type II neurons to assess whether the electrophysiological trends that we observed be- 
tween these two classes could be corroborated. From this analysis we found that the basal type II Kv1.1 antibody staining was significantly lower than the apical type II antibody staining ( $p>0.05)$, substantiating the differences in accommodation that we had observed electrophysiologically (Fig. 7B).

\section{Discussion}

Although the type II spiral ganglion neurons only make up a small proportion of the spiral ganglion, they are likely to have a profound effect on neural encoding because of their extensive peripheral innervation patterns (Spoendlin, 1973; Perkins and Morest, 1975; Berglund and Ryugo, 1987; Ryugo, 1992). In contrast to the oneto-one synaptic connections made between the type I neurons and inner hair cells, each type II neuron receives synaptic input from 15-20 outer hair cells (Spoendlin, 1972). Classically, dual innervation patterns such as those displayed in the cochlea are designed to enhance coding of two separate aspects of a sensory signal. One-to-one peripheral innervation, such as that exhibited by the type I neurons, is designed to convey sensory information with high resolution, often at suprathreshold levels. Thus, precise innervation of the sensory receptors retains the specificity of the peripheral receptor organization, which in the case of the auditory system is organized according to frequency. The convergent innervation pattern displayed by the type II neurons, on the other hand, presumably enhances sensitivity at the expense of resolution. On the basis of these peripheral innervation patterns, one might hypothesize that the two classes of primary afferents in the cochlea process sound stimuli in very different ways.

Type I neuronal response properties clearly reflect their precise innervation, as evidenced by their high selectivity for a limited range of sound frequencies at low stimulus levels (Kiang et al., 1965; Liberman, 1978). A high degree of sensitivity predicted by the profuse innervation patterns of the type II neurons, however, has not been established. Nevertheless, indirect approaches have yielded some pertinent information. Reduction of the efficacy of the outer hair cells by stimulating their inhibitory efferent supply decreased the sensitivity of type I neurons to sound stimuli, resulting in rate-level functions shifted to higher stimulus intensities, depressed spontaneous firing rates, and increased thresholds at characteristic frequency (Guinan and Gifford, 1988a,b,c). These effects are generally accepted to be caused by alteration of the mechanical properties of the outer hair cells (Brownell et al., 1985) that affect the mechanical tuning of the basilar membrane, thus indirectly affecting the sensitivity of the inner hair cell-type I responses to sound (Dallos and Corey, 1991; Holley, 1996). Whatever the mechanism, however, these classic experiments demonstrate that the outer hair cell-neural system enhances the responsiveness of the type I neurons to sound.

This view has been brought into question, however, by the limited data resulting from numerous attempts to record from type II neurons in vivo. To date, only one successful in vivo recording with morphological confirmation has been reported (Robertson, 1984). The neuron in this study, however, did not
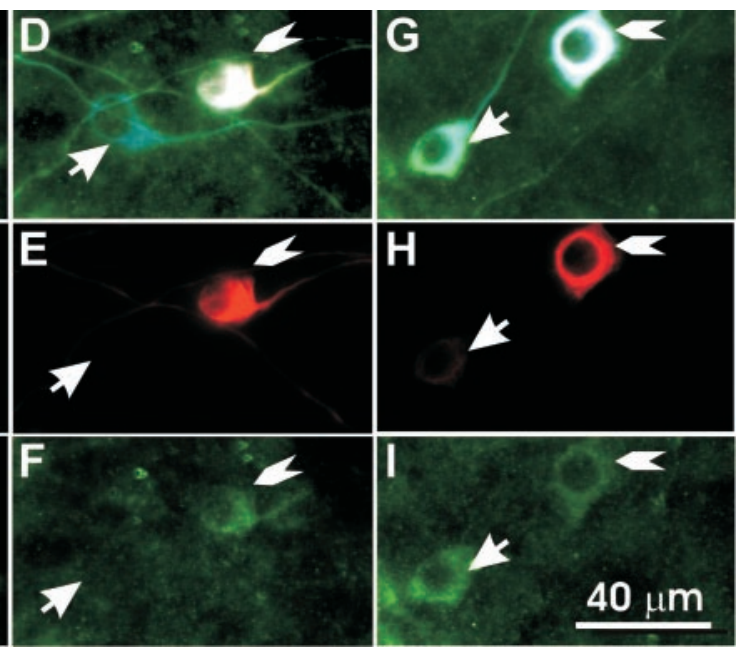

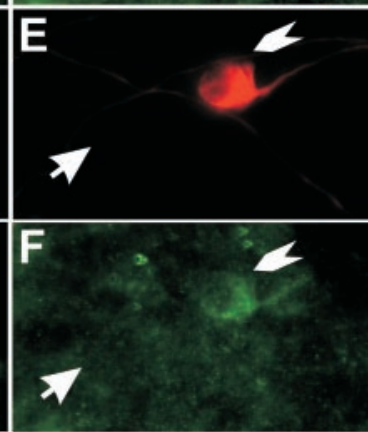

Figure 6. Basal and apical type II neurons show contrasting $\alpha$-Kv1.1 staining patterns when compared with neighboring type spiral ganglion neurons. $A-C$, Spiral ganglion neurons isolated from the basal cochlea were stained with $\alpha$ - $\beta$-tubulin (blue) ( three antibodies. $H$, Fluorescent image of $\alpha$-peripherin staining. I, Fluorescent image of $\alpha$-Kv1.1 staining.
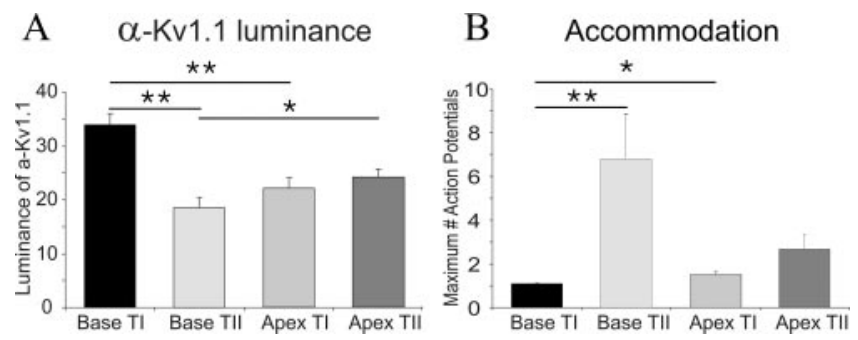

Figure 7. Kv1.1 antibody distribution showed patterns that were consistent with the electrophysiological parameter of accommodation. A, Quantitative assessment of the Kv1.1 luminance of the type I and type II spiral ganglion neurons taken from the apical and basal cochlea. $B, A P_{\max }$ measurements from Figure $3 A$ reformatted for the purpose of comparison.

fire action potentials. Whether this was indicative of a cell that was unresponsive to sound or one that had been damaged by electrode penetration could not be determined. Others have found similar high-threshold neurons in the spiral ganglion that also showed delayed responses to sound (Brown, 1994; Robertson et al., 1999). The investigators hypothesized that they may represent the firing patterns of the unmyelinated type II neurons. They further speculated that if these recordings did in fact represent type II neurons, then they may mediate sensitivity to highthreshold stimuli such as painful stimuli, analogous to the $\mathrm{C}$ fibers in the somatosensory system (Brown, 1994). Nevertheless, this issue is still unresolved because attempts to fill these highthreshold neurons with dye to determine their peripheral innervation were unsuccessful. The morphological identity of the high-threshold neurons thus remains an open question, as does the precise modality conveyed by the type II neurons.

Because the endogenous membrane properties of neurons are tailored, in general, to their functional roles (Llinás, 1988; Levitan and Kaczmarek, 1997; Hille, 2001), examination of the firing features of the spiral ganglion neurons may lend important clues about their function. By using a marker for a type II-specific 
protein, we were able to study the type I and type II neuronal populations separately. The analysis of type I spiral ganglion neurons confirmed previous work from our laboratory showing that their intrinsic properties differed according to region of innervation (Adamson et al., 2002b). Neurons isolated from the basal cochlea showed rapid accommodation and fast kinetics, whereas neurons isolated from the apical cochlea showed varied accommodation and slower kinetics. In this study, we were able to establish that these frequency-specific differences could be attributed to the type I neurons alone, having removed type II neurons from the analysis.

When considered on their own, type II neurons systematically showed slow kinetics and low action potential thresholds. The greatest differences between the type I and type II spiral ganglion neurons were between those isolated from the basal cochlea. Exponentials fitted to the onset of the subthreshold depolarizations, the decay of the fast AHP at threshold, and the inward rectification in addition to the action potential latencies and durations were all significantly prolonged in the type II neurons, thus displaying a markedly slow phenotype in a small population of neurons that innervate the outer hair cells of the high-frequency region of the cochlea. Although the apical type II neurons also showed many of these same features, only the action potential latencies, durations, and time course of the fast afterhyperpolarizations differed significantly from the apical type I neurons. Despite the fact that electrophysiological threshold was significantly different only between the basal type I and II neurons, the magnitude of the inward rectification differed significantly from the type I neurons for both apical and basal type II spiral ganglion neurons. Because the inward rectification is caused by $I_{\mathrm{h}}$ currents, which have been identified in the spiral ganglion neurons (Mo et al., 2002), and the relationship between the $I_{\mathrm{h}}$ currents and threshold has been convincingly established by others (Pape and McCormick, 1989; McCormick and Huguenard, 1992; Erickson et al., 1993), we speculate that it may also play a role in regulating the electrophysiological thresholds in spiral ganglion neurons.

Analysis of the five basal and three apical type II spiral ganglion neurons showed that none of the electrophysiological parameters that we measured were significantly different between type II neurons isolated from different cochlear regions. Nevertheless, it is obvious that the physiological parameters measured from basal type II neurons were slower (i.e., prolonged latency, less accommodation) than those measured from apical type II neurons. Furthermore, when recordings were made from pairs or clusters of cells in which type II neurons were identified, marked differences were evident in the electrophysiological responses of neighboring type I and type II neurons. These differences were also evident when Kv1.1 antibody staining density was assessed in neurons isolated from the apex and the base. The differential type I/II staining intensity in each of the regions and the opposite intensities when basal type II neurons were compared with apical ones substantiate our electrophysiological observations. This suggests the intriguing possibility that the kinetic features of the type II spiral ganglion neurons, although skewed to slower kinetics, are oriented as a mirror image to that established for the type I spiral ganglion neurons.

The complexity of the heterogeneous firing features outlined above, however, should not overshadow the basic observation that the endogenous properties of the type I spiral ganglion neurons, in general, show faster kinetics and more rapid accommodation than their type II counterparts. This is exemplified most clearly for basal type I and type II spiral ganglion neurons. The rapid accommodation and kinetics of the type I neurons, along with their one-to-one synaptic connections with inner hair cell sensory receptors, support their role in establishing the precise frequency coding and timing that is a hallmark of the auditory system.

Interestingly, a similar physiological-anatomical relationship can also be found in higher auditory centers. For example, the bushy cells in the cochlear nucleus and the principal cells in the medial nucleus of the trapezoid body receive synaptic input from very few neurons, and both are characterized by rapid accommodation (Oertel et al., 1988; Brew and Forsythe 1995). Conversely, the stellate cells of the cochlear nucleus receive profuse synaptic connections and display slow accommodation (Oertel et al., 1988). Both centrally and peripherally, the low-threshold $\mathrm{K}^{+}$ currents that contribute to neuronal accommodation (Manis and Marx, 1991; Brew and Forsythe 1995; Rathouz and Trussell, 1998; Mo et al., 2002; Brew et al., 2003) appear to represent an intrinsic specialization that contributes to the exquisite temporal resolution observed in certain auditory nuclei (Oertel, 1997; Trussell, 1999).

The purpose for performing the experiments herein was to gain insights into the functional relevance of the type II neurons by analyzing their endogenous firing features. The observed electrophysiological diversity between the two classes of primary auditory neurons argues for different roles for the type I and II neurons. In contrast to the type I spiral ganglion neurons, the type II neurons show markedly distinct kinetics and receive a classically convergent innervation pattern that is also present in other sensory systems. Like the rod neural circuitry in the retina, the type II spiral ganglion neurons appear to be designed to integrate input from the many receptors that they innervate. This would have very little impact, however, on signal detection without the process of temporal summation. The slow kinetics of the type II spiral ganglion neurons, along with their ability to respond at relatively hyperpolarized voltage levels, are features consistent with mechanisms designed to detect low-intensity stimuli.

\section{References}

Adamson CL, Reid MA, Davis RL (2002a) Opposite actions of brainderived neurotrophic factor and neurotrophin-3 on firing features and ion channel composition of murine spiral ganglion neurons. J Neurosci 22:1385-1396.

Adamson CL, Reid MA, Mo ZL, Bowne-English J, Davis RL (2002b) Firing features and potassium channel content of murine spiral ganglion neurons vary with cochlear location. J Comp Neurol 447:331-350.

Berglund AM, Ryugo DK (1987) Hair cell innervation by spiral ganglion neurons in the mouse. J Comp Neurol 255:560-570.

Brew HM, Forsythe ID (1995) Two voltage-dependent $\mathrm{K}^{+}$conductances with complementary functions in postsynaptic integration at a central auditory synapse. J Neurosci 15:8011-8022.

Brew HM, Hallows JL, Tempel BL (2003) Hyperexcitability and reduced low threshold potassium currents in auditory neurons of mice lacking the channel subunit Kv1.1. J Physiol (Lond) 548:1-20.

Brown MC (1994) Antidromic responses of single units from the spiral ganglion. J Neurophysiol 71:1835-1847.

Brownell WE, Bader CR, Bertrand D, de Ribaupierre Y (1985) Evoked mechanical responses of isolated cochlear outer hair cells. Science 227:194-196.

Dallos P, Corey ME (1991) The role of outer hair cell motility in cochlear tuning. Curr Opin Neurobiol 1:215-220.

Erickson KR, Ronnekleiv OK, Kelly MJ (1993) Electrophysiology of guineapig supraoptic neurones: role of a hyperpolarization-activated cation current in phasic firing. J Physiol (Lond) 460:407-425.

Fanning AS, Anderson JM (1999) Protein modules as organizers of membrane structure. Curr Opin Cell Biol 11:432-439.

Guinan JJ, Gifford ML (1988a) Effects of electrical stimulation of efferent olivocochlear neurons on cat auditory-nerve fibers. I. Rate-level functions. Hear Res 33:97-114. 
Guinan JJ, Gifford ML (1988b) Effects of electrical stimulation of efferent olivocochlear neurons on cat auditory-nerve fibers. II. Spontaneous rate. Hear Res 33:115-128.

Guinan JJ, Gifford ML (1988c) Effects of electrical stimulation of efferent olivocochlear neurons on cat auditory-nerve fibers. III. Tuning curves and thresholds at CF. Hear Res 37:29-46.

Hafidi A (1998) Peripherin-like immunoreactivity in type II spiral ganglion cell body and projections. Brain Res 805:181-190.

Hille B (2001) Ionic channels of excitable membranes. Sunderland, MA: Sinauer.

Holley MC (1996) Outer hair cell motility. In: The cochlea (Dallos P, Popper AN, Fay RR, eds), pp 386-434. New York: Springer.

Hsu Y, Firestein BL, Davis RL (2002) Differential distribution of membrane-associated guanylate kinases (MAGUKs) in type I and type II spiral ganglion neurons. Assoc Res Otolaryngol 25:410.

Kiang NYS, Watanabe T, Thomas EC, Clark LF (1965) Discharge patterns of single fibers in the cat's auditory nerve. Cambridge, MA: MIT.

Koch RO, Wanner SG, Koschak A, Hanner M, Schwarzer C, Kaczorowski GJ, Slaughter RS, Garcia ML, Knaus HG (1997) Complex subunit assembly of neuronal voltage-gated $\mathrm{K}^{+}$channels. Basis for high-affinity toxin interactions and pharmacology. J Biol Chem 272:27577-27581.

Levitan IB, Kaczmarek LK (1997) The neuron. New York: Oxford UP

Liberman MC (1978) Auditory-nerve response from cats raised in a lownoise chamber. J Acoust Soc Am 63:442-455.

Llinás RR (1988) The intrinsic electrophysiological properties of mammalian neurons: insights into central nervous system function. Science 242:1654-1664.

Magistretti J, Mantegazza M, Guatteo E, Wanke E (1996) Action potentials recorded with patch-clamp amplifiers: are they genuine? Trends Neurosci 19:530-534.

Manis PB, Marx SO (1991) Outward currents in isolated ventral cochlear nucleus neurons. J Neurosci 11:2865-2880.

McCormick DA, Huguenard JR (1992) A model of the electrophysiological properties of thalamocortical relay neurons. J Neurophysiol 68:1384-1400.

Mo Z-L, Davis RL (1997a) Endogenous firing patterns of murine spiral ganglion neurons. J Neurophysiol 77:1294-1305.

Mo Z-L, Davis RL (1997b) Heterogeneous voltage dependence of inward rectifier currents in spiral ganglion neurons. J Neurophysiol 78:3019-3027.

Mo ZL, Adamson CL, Davis RL (2002) Dendrotoxin-sensitive K(+) currents contribute to accommodation in murine spiral ganglion neurons. J Physiol (Lond) 542:763-778.
Mou K, Adamson CL, Davis RL (1998) Time-dependence and cell-type specificity of synergistic neurotrophin actions on spiral ganglion neurons. J Comp Neurol 402:129-139.

Oertel D (1997) Encoding of timing in the brain stem auditory nuclei of vertebrates. Neuron 19:959-962.

Oertel D, Wu SH, Hirsh JA (1988) Electrical characteristics of cells and neuronal circuitry in the cochlear nuclei studied with intracellular recordings from brain slices. In: Auditory function: neurological bases of hearing (Edelman GM, Gall WE, Cowan WM, eds), pp 313-336. New York: Wiley.

Pape H-C, McCormick DA (1989) Noradrenaline and serotonin selectively modulate thalamic burst firing by enhancing a hyperpolarizationactivated cation current. Nature 340:715-718.

Perkins RE, Morest DK (1975) A study of cochlear innervation patterns in cats and rats with the Golgi method and Nomarkski optics. J Comp Neurol 163:129-158.

Pujol R, Lavigne-Rebillard M, Lenoir M (1998) Development of sensory and neural structures in the mammalian cochlea. In: Development of the auditory system (Rubel EW, Popper AN, Fay RR, eds), pp 146-192. New York: Springer-Verlag.

Rathouz M, Trussell L (1998) Characterization of outward currents in neurons of the avian nucleus magnocellularis. J Neurophysiol 80:2824-2835

Reid MA, Davis RL (2002) Characterization of peripherin-positive spiral ganglion neurons. Assoc Res Otolaryngol 25:411.

Reid MA, Davis RL (2003) Firing properties of putative type II spiral ganglion neurons in vitro. Assoc Res Otolaryngol 26:784.

Robertson D (1984) Horseradish peroxidase injection of physiologically characterized afferent and efferent neurones in the guinea pig spiral ganglion. Hear Res 15:113-121.

Robertson D, Sellick PM, Patuzzi R (1999) The continuing search for outer hair cell afferents in the guinea pig spiral ganglion. Hear Res 136:151-158.

Romand MR, Romand R (1987) The ultrastructure of spiral ganglion cells in the mouse. Acta Otolaryngol 104:29-39.

Ryugo DK (1992) The auditory nerve: peripheral innervation cell body morphology, and central projections. In: The mammalian auditory pathway: neuroanatomy (Popper AN, Fay RR, eds), pp 34-93. New York: Springer.

Spoendlin H (1972) Innervation densities of the cochlea. Acta Otolaryngol $73: 235-248$

Spoendlin H (1973) The innervation of the cochlea receptor. In: Mechanisms in hearing (Møller AR, ed), pp 185-229. New York: Academic.

Trussell LO (1999) Synaptic mechanisms for coding timing in auditory neurons. Annu Rev Physiol 61:477-496. 